\title{
MATHEMATICAL MODEL, COMPUTER AIDED DESIGN AND PROGRAMMING OF A MULTIFUNCTIONAL FLYING OBJECT
}

\author{
Ernest Czerwiński ${ }^{1}$, Michał Szewc ${ }^{1}$, Igor Wojtunik ${ }^{1}$, Jan Awrejcewicz ${ }^{2}$, Paweł Olejnik ${ }^{2}$ \\ ${ }^{1}$ Faculty of Mechanical Engineering, Lodz University of Technology, \\ 1/15 Stefanowski Str., 90-924 Łódź, Poland \\ ${ }^{2}$ Department of Automation and Biomechanics, Lodz University of Technology, Eódź, Poland \\ E-mail: pawel.olejnik@p.lodz.pl (corresponding author)
}

Received 11 November 2013; accepted 28 February 2014
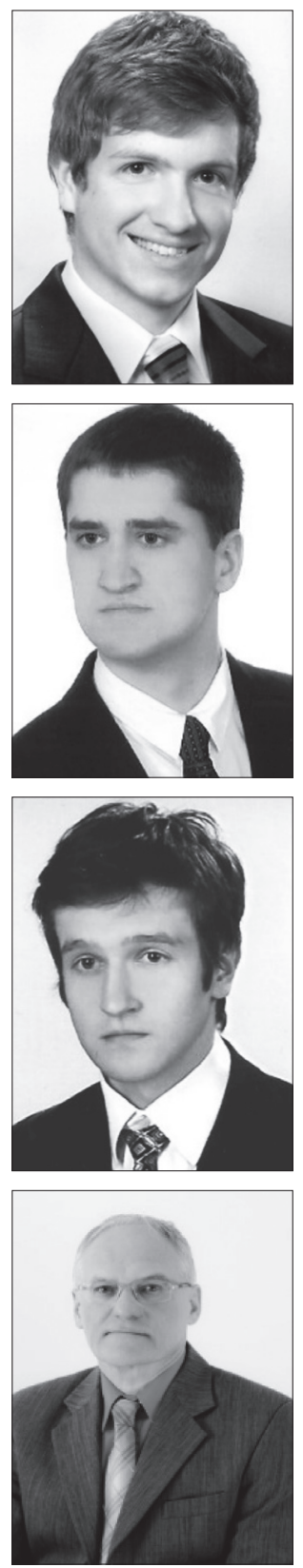

Ernest CZERWIŃSKI, MSc.

Education: 2009-2014 - student at the Faculty of Mechanical Engineering of Lodz University of Technology, second degree studies in Mechatronics. Research interests: classical mechanics, theory of control, mechatronics, CAD design, system modelling, numerical simulations.

Michał SZEWC, $M S c$.

Education: 2009-2014 - student at the Faculty of Mechanical Engineering of Lodz University of Technology, second degree studies in Mechatronics.

Research interests: classical mechanics, theory of control, mechatronics, CAD design, system modelling, numerical simulations.

\section{Igor WOJTUNIK, MSc.}

Education: 2009-2014 - student at the Faculty of Mechanical Engineering of Lodz University of Technology, second degree studies in Mechatronics.

Research interests: classical mechanics, theory of control, mechatronics, CAD design, system modelling, numerical simulations.

\section{Jan AWREJCEWICZ, Prof.}

Education: MSc and PhD degrees in the field of Mechanics from Lodz University of Technology in 1977 and 1981; Bachelor's degree in Philosophy - in 1978 from the University of Lodz, and DSc degree in Mechanics from Lodz University of Technology in 1990. Affiliations and functions: in 1994 he received the title of Professor from the President of Poland, Lech Watesa, and in 1996 he obtained the golden cross of merit from the next President of Poland, Aleksander Kwaśniewski. He is a contributor to 50 different research journals and to 300 conferences.

Research interests: mathematics, mechanics, biomechanics, automatics, physics and computer oriented sciences. 
Publications: author or co-author of 630 publications in scientific journals and conference proceedings, monographs (43), text books (2), edited volumes (9), conference proceedings (11), journal special issues (15), other books (9) and other short communications and unpublished reports (284).

Present position: Head of Department of Automatics and Biomechanics, and the Head of PhD School on 'Mechanics' associated with the Faculty of Mechanical Engineering of Lodz University of Technology.

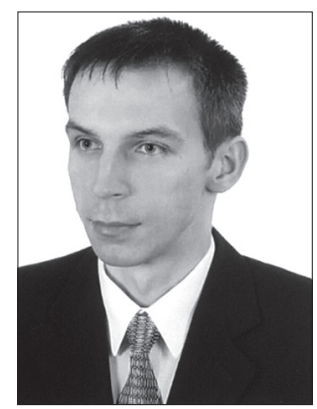

\title{
Pawel OLEJNIK, Assistant Prof.
}

Education: in 2000 - MSc degree in Computer Physics from the Faculty of Technical Physics, Computer Science and Applied Mathematics at Lodz University of Technology. In 2002 - doctoral thesis with a distinction of experimental research and obtained a Doctorate in Technical Sciences. In 2013 - DSc degree in Applied Mechanics from Lodz University of Technology. Functions: research and teaching job in the Department of Automation, Biomechanics and Mechatronics at the Faculty of Mechanical Engineering.

Research interests: computational techniques for solving discrete dynamics problems, experimental research on laboratory stations, active control of nonlinear systems, analysis and modelling of mechatronic systems.

\begin{abstract}
The quadrocopter, an unmanned aerial vehicle (UAV), is a type of aerodynamic object using thrust generated by the propellers revolving around the rotor mast. The main areas of focus for complete design and engineering research should be on its stabilization and realization of tasks to be performed during flight. To accomplish these tasks, it is necessary to consider the following stages: modeling of motion dynamics, computer-aided design, programming of the control unit, precise implementation, carefully selected motors and sensors, i.e. a gyroscope, accelerometer, camera, communication module, GPS module, and others. The presented results of research and experiments carried out at Lodz University of Technology in the Department of Automation and Biomechanics take into consideration most of these issues.
\end{abstract}

Keywords: unmanned aerial vehicle, quadrotor, mathematical modeling, computer aided design.

\section{Introduction}

A modern quadrotor helicopter, also referred to as a quadrocopter, is an unmanned aerial vehicle (UAV). Due to the availability of high-speed brush-less motors, inertial measurement units based on MEMS technology and high power to weight ratio lithium-polymer (LiPo) batteries (170W/Kg and more), unmanned quadrocopters can now be designed and fabricated in various ways, but their control is still complicated. In the last decade, many researchers have put a lot of effort in this area. Selected concepts of numerical modelling and real realizations are provided in the introduction.

Classification of miscellaneous types of unmanned vehicles has been synthesized in (Gupte et al. 2012) based on the following features: the input and output parameters, mechanism of flying, control systems, dynamic models and sensors. The proposed vehicle construction is based on a typical quadrotor model with an inertial measurement unit, sensors, electronic speed controllers with the microcontroller at the centre and four rotors at each end. Such an approach to the construction of many types of quadrotors constitutes, in general, the works of young engineers or even more advanced researchers.

In most cases, a quadrocopter is proved to be a very unstable vehicle. This is conditioned by its under-actu- ated behaviour. Therefore, different control techniques must be utilized to achieve controlled take off, flight and landing of the sensitive aerial vehicle. Algorithms like PID (Bi, Duan 2013), backstepping (Yali et al. 2012), Kalman filtering (Zhang el al. 2011), and feedback linearization (Ryll et al. 2012) have mainly been used and are proved to work well with a quadrocopter.

\subsection{Numerical simulations of a quadrotor UAV}

Numerical models are an important part in the design of real aerial vehicles. One can test any mathematical models and control strategies available in the wide range of control theory. The design and control of an ideal quadrotor system has been proposed in (Senkul, Altug 2013). Authors of the contribution propose an alternative propulsion system formed by tilting rotors. This novel design eliminates the need of tilting the airframe and suggests superior performance with respect to the regular quadrotor design. The mathematical model of the tilt-roll quadrotor and the designed control algorithms have been explained. On the basis of a numerical simulation carried out in MATLAB, a comparison of the proposed system with the regular quadrotor indicates better performance.

In the paper by K. Runcharoon and V. Srichatrapimuk (2013), the problem of attitude control with 
external disturbances has been considered using nonlinear sliding mode control (Olejnik, Awrejcewicz 2013) with two difference switching functions. The sliding mode controller is able to drive a quadrotor to the desired position and yaw. Comparisons shown in the 2- and 3-dimensional graphs exhibit the ability of this approach to stabilize the quadrotor and move it to any desired position despite any existing disturbances.

Dynamics and kinematics of a quadrotor aerial vehicle were analysed in (Yali et al. 2012). The aim of a nonlinear dynamic model was to attain the best control algorithm. Therefore, the whole system was divided into three interconnected parts: attitude subsystem, vertical subsystem and position subsystem. Then, nonlinear control strategies such as SDRE and backstepping were introduced. Various models implementing control laws stabilizing the quadrotor aerial vehicle with good tracking performance and robustness of the whole control system were investigated.

A novel actuation concept for a quadrotor unmanned aerial vehicle (UAV) has been presented by M. Ryll et al. (2012). Propellers of the UAV's numerical model are allowed to tilt about the axes connecting them to the main body's frame. Based on simulations, the additional actuation of four degrees-of-freedom gains the full controllability over the quadrotor position and orientation, resulting in a fully controlled rigid flying body. After deriving the dynamical model of the proposed quadrotor, its controllability properties were discussed. Using a dynamic feedback linearization technique, a nonlinear trajectory tracking controller was proposed.

In (Minh, Ha 2010), another interesting mathematical model of a quadrotor using Lagrange equation was analysed. In this study, the authors introduce some vision algorithms of pose and motion estimation using a two-camera system. With regard to stabilization of the attitude of the quadrotor's model and measurement noise rejection, both a Linear Quadratic (LQ) tracking controller with integral action and an optimal Linear Quadratic Gaussian (LQG) control with integral action as well have been designed in MATLAB. This paper demonstrates the usefulness of such a control.

The decentralized tracking controller has been designed in (Mohammadi, Mohammad Shahri 2013) based on a model reference adaptive control for the general form of a UAV. Outer kinematic position control loops and inner dynamic attitude control loop create two stages of the incorporated control strategy. The simulation results showed that the designed controllers are robust and guarantee good trajectory tracking in the presence of parametric and nonparametric uncertainties. These controllers are also able to reject sustained disturbances, but the drawback of this method is that uncertainty in control input and measurement noises is not considered.
In the study by G. V. Raffo et al. (2011), two techniques are combined and adapted for a quadrocopter in order to synthesize the inner-loop controller. In the outer loop, an integral predictive controller is designed by determining the roll and pitch reference angles for the inner-loop controller. The outer-loop controller with integral action is added to perform the sustained disturbance rejection. The integral term of the $x y$ position error has been included in the error vector. The robust performance provided by the inner-outer control strategy has been tested by simulation confirming excellent tracking performance achieved with the proposed control structure.

\subsection{Design and control of prototype quadrotors}

The modelling of a quadrotor UAV is always associated with the application of a control technique that takes into consideration the type of sensors and the method of communication with the flying object. This provides real solutions, some of which are cited below.

Theoretical models of a quadrotor's aerodynamics with non-zero free-stream velocities based on helicopter momentum and blade element theory validated by static tests and flight data have been presented in (Hoffmann et al. 2011). Controllers are derived using these models and implemented on the quadrotor helicopter; they demonstrate significant improvements over existing methods. This work describes the vehicle's dynamics, design, and control systems with flight demonstrations of successful completion of autonomous trajectory tracking.

Construction of a quadrotor prototype which would allow testing the approximate adaptive methods for quadrotor control has been considered in (Nicol et al. 2011). The Cerebellar Model Arithmetic Computer (CMAC) algorithm provides a computationally-efficient and accurate nonlinear approximator. Authors propose a robust method that relies on an alternate set of weights to guide the training of the weights used in the controller CMAC. This paper describes the design of a prototype helicopter suitable for testing the control method. In the experiment, the new method stops weight drift during a shake test and adapts on-line to a significant added payload.

The study by K. Alexis et al. (2012) addresses the control problem of an unmanned quadrotor that manoeuvres in an indoor environment and with absence of absolute localisation data. Based on several components such as an attached inertia measurement unit, a sonar and an optic-flow sensor, the state vector is estimated using sensor fusion algorithms. A novel switching model predictive controller is designed in order to achieve precise trajectory control under the influence of wind gusts. The proposed controller algorithm accounts for the state and actuation constraints of the system. The experimental results indicate the overall system's efficiency in carrying out position/altitude/attitude set-point manoeuvres. 
An autonomous visual tracking and landing system for a low-cost unmanned quadrotor has been proposed by Y. Bi, H. Duan (2013). The designed hybrid system demonstrates the use and fast deployment ability of miniature quadrotors. The adopted computer vision algorithms are rather simple, fast and effective under different lighting conditions. The quadrotor is controlled with a classical PID controller for autonomous visual tracking and landing on a moving carrier. Rich vision information can guarantee excellent performance of the designed quadrotor's tracking and landing system.

A fully autonomous quadrotor in a heterogeneous air-ground multi-robot system was established in (Zhang et al. 2011) using two on-board sensors: a monocular camera and inertial measurement units (IMUs). An extended continuous-discrete Kalman filter is applied, in which high-frequency IMU data drive the prediction, while estimates are corrected by accurate and steady vision data. High-frequency fusion at $100 \mathrm{~Hz}$ is achieved. Moreover, time delay analysis and data synchronizations are conducted to further improve the pose/motion estimation of the quadrotor. The complete on-board implementation of sensor data processing and control algorithms reduces the influence of data transfer time delay, enables autonomous task accomplishment and extends operation space. Higher pose estimation accuracy and smaller control errors compared to standard work are achieved in real-time hovering and tracking experiments.

The survey paper by D. Sahoo et al. (2010) presents a mission-centric approach to controlling the optical axis of a video camera mounted on a camera manipulator and fixed to a quadrotor remotely operated vehicle. A 4-dof quadrotor UAV model is combined with a 2-dof camera kinematic model. The whole system provides full 6-dof actuation of the camera view. In this approach, all signals are described in the camera frame. The closed-loop controller is designed on the basis of a Lyapunov-type analysis. Computer simulation results are provided in MATLAB to demonstrate the elaborated controller.

In the works reviewed previously, a lot of attention is paid to the control and the numerical simulations. Our work extends these issues related to the dynamical analysis and control of the unmanned aerial vehicle by illustrating its design (FEM analysis of arms and main platform), estimation of unknown parameters and the design and programming of an electronic control unit.

\section{Computer aided design (CAD) of the aerial vehicle}

A quadrocopter as an unmanned aerial vehicle is a type of aerodynamic object using thrust generated by propellers revolving around the rotor mast (Fig. 1).

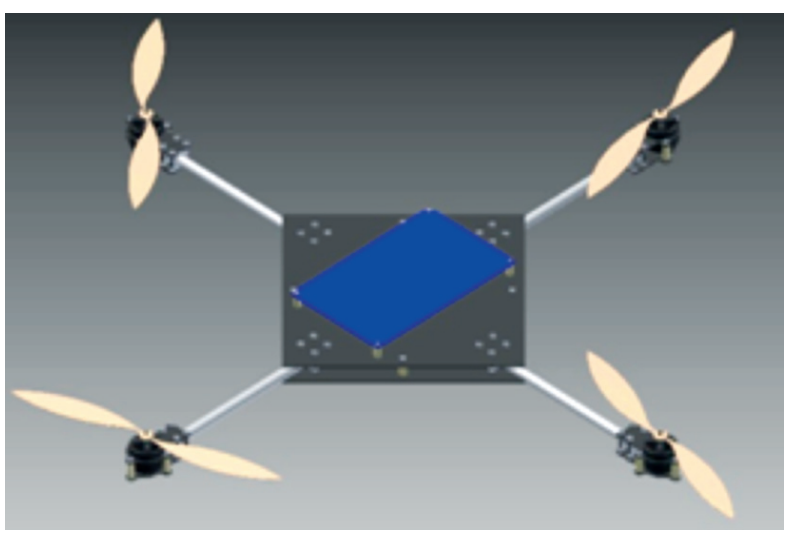

Fig. 1. The quadrocopter's CAD in inventor

An aluminum tube with an outside diameter of $10 \mathrm{~mm}, 8 \mathrm{~mm}$ inner diameter and $180 \mathrm{~mm}$ length has been used to create a single arm. The tube is made of aluminum alloy PA6 and weighs $14 \mathrm{~g}$. A strength analysis of this element has been performed. The tube was attached to the platform using specially designed mounting brackets shown in figure 2 .

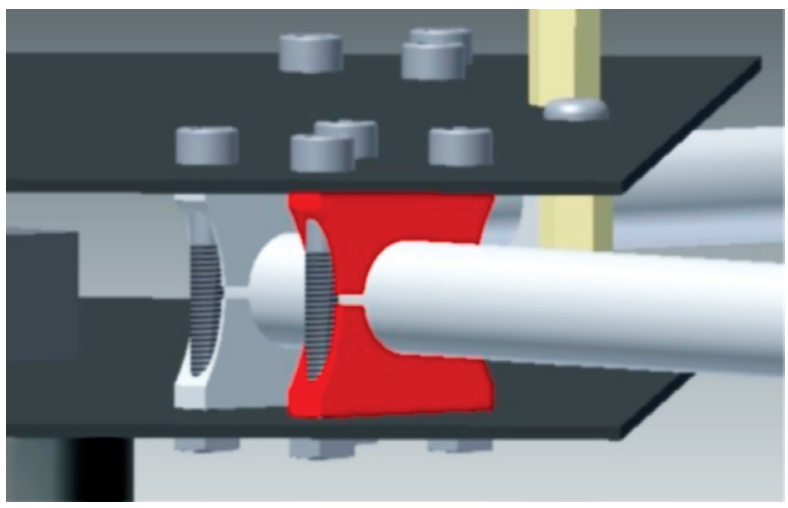

Fig. 2. The brackets fixing the arm to the quadrocopter's platform

Greatest stresses occur in the arms of the quadrocopter as shown in figure $3 \mathrm{a}$ and in the corners of the platform. Therefore, both plates composing the platform are made of a light composite denoted by the G10 symbol (NEMA norm). It is visible in figures $3 \mathrm{~b}$ and $3 \mathrm{c}$.

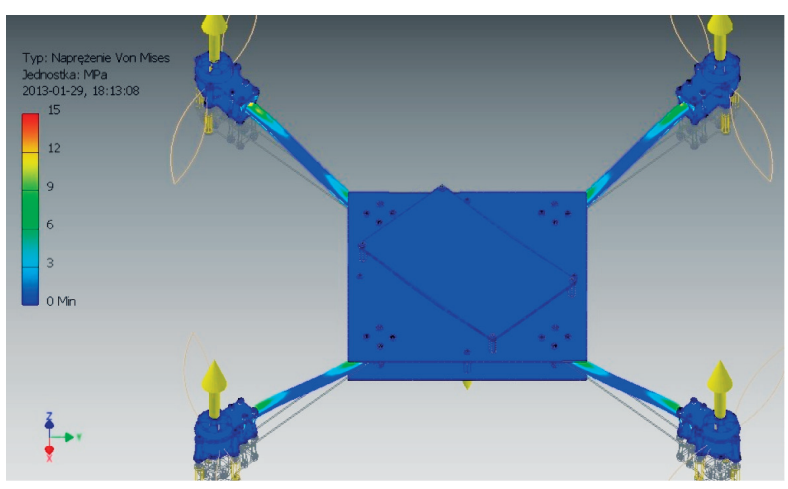

(a) 


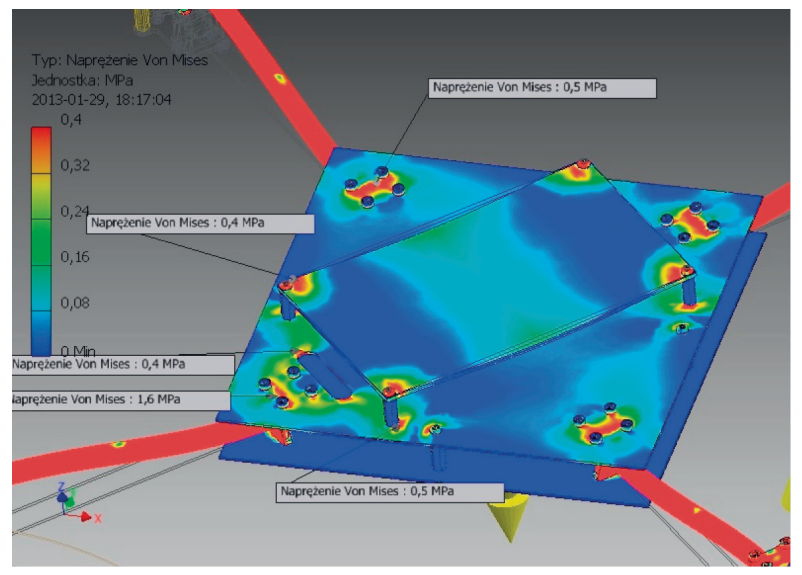

(b)

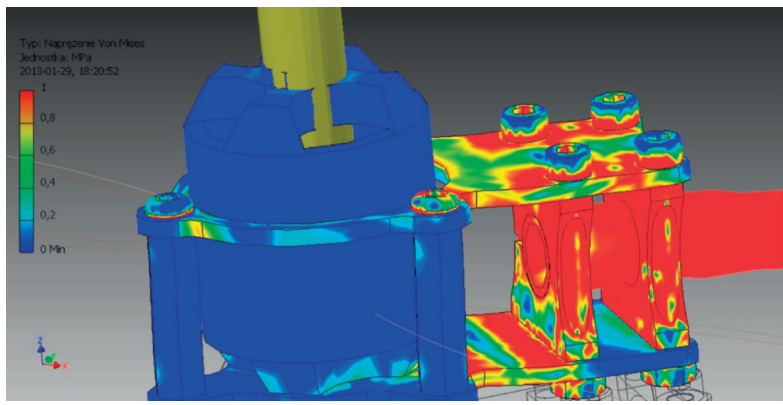

(c)

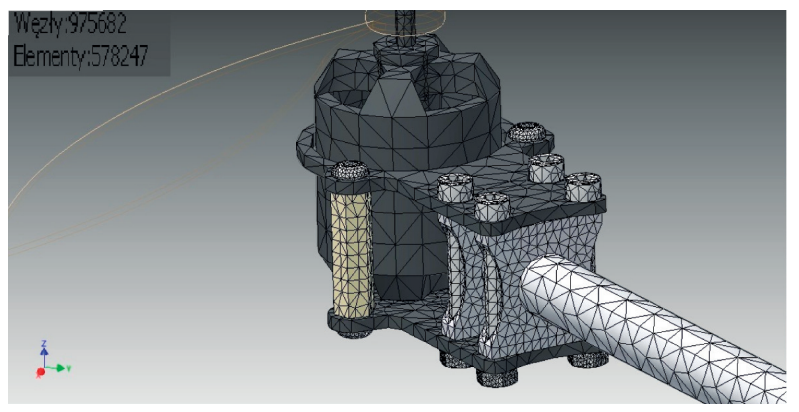

(d)

Fig. 3. Stress analysis of the quadrocopter's: arms (a); main platform with additional plate for mounting electronics (b); basket mounting the engine (c); and its FEM discretization (d)

Basically, the prototype design consists of three main parts: the platform, four arms and four engine mounting brackets. The parts during flight are illustrated in figure 4 .

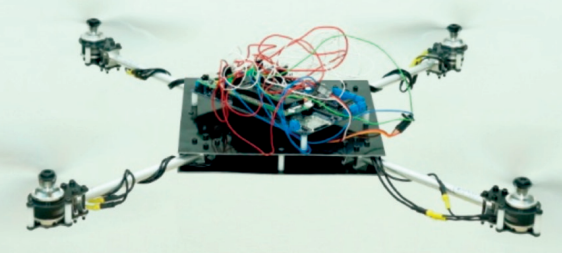

Fig. 4. The prototype quadrocopter
According to the information provided by the manufacturer of propellers, and on the basis of measurements made by the authors of the study, the maximum thrust generated by an engine (an option with big propellers) is about $10 \mathrm{~N}$. Therefore, the total lifting force (thrust) of the entire construction with four engines is $40 \mathrm{~N}$, which directly determines that the maximum weight of the quadrocopter in order to become airborne is $4 \mathrm{~kg}$. However, the thrust at $40 \mathrm{~N}$ is achieved at the maximum motor speed and the ability to control the structure weighting $4 \mathrm{~kg}$ is close to zero. The thrust depends on many parameters of the motors and propellers. In the most optimal case, it should be at least 50\% larger than the weight of the whole structure (including all elements of the propulsion and control unit). To maintain a margin of weight for future development and better control of the quadrocopter, the total weight should not exceed $1.5 \mathrm{~kg}$.

Another important issue, which should be noted, is the very high frequency of vibrations generated by the motors. Due to this, imbalance in the motor that produces the vibrations transmitted to the structure occurs. Moreover, the frequency and amplitude of these vibrations are difficult to determine, because their shape is affected by the variable speed of the AC motors. In addition, the propellers used in the project are made of plastic so that they would be sufficiently flexible. While spinning at speeds that exceed $10000 \mathrm{rev} / \mathrm{min}$, under the influence of changing air masses acting on them, they also contribute to the increase of occurring flight vibrations. To prevent the transmission of vibrations to the core, where the controls and electronics are placed, proper materials that are not conducive to the propagation of vibration are selected. There are also geometric restrictions resulting from the propulsion and steering elements. As previously mentioned, we were guided in the design by two basic conditions, i.e. light weight and economy.

\section{Estimation of model parameters}

The model is made in inventor, which enables the analysis of the dynamic properties of the whole structure. Particularly important for the project is the ability to read some of the necessary control parameters. One of them is the moments of inertia about the axes of a coordinate system. The analytical calculation of the moments of inertia of the structure with such complicated geometry is a very complex issue requiring a lot of work, and the results would be unsatisfactory.

\subsection{Structural properties}

It is trivial to obtain these parameters directly from the modeled structure in Inventor and more accurate results than those obtained by using the method of 
discretization are determined. On this basis, the moments of inertia with respect to the axes $O X, O Y, O Z$ of the structure are as follows:

$I_{x x}=0.173, I_{y y}=0.0169, I_{z z}=0.0333\left[\mathrm{~kg} \cdot \mathrm{m}^{2}\right]$.

Another significant advantage is the ability to read the position of the center of gravity of the whole structure. There is always a certain inaccuracy resulting from the lack of modeled wiring, but their weight is negligible compared to the rest of the structure. The structure's centre of gravity is placed at the point:

$$
X=0.001, Y=0.09, Z=12.475 \text { [mm]. }
$$

To implement the control system it is also necessary to read the moment of inertia of the propeller:

$$
I_{p}=1.8 \cdot 10^{-5}\left[\mathrm{~kg} \cdot \mathrm{m}^{2}\right] .
$$

The main parameter that can be determined by the program is the total mass of the quadrocopter's structure, which amounts to $m=1.096 \mathrm{~kg}$ with all propulsion systems and electronics (Tab.).

Table. Weight of basic quadrocopter elements

$\begin{array}{lc}\text { Element } & \text { Weight }[\mathrm{kg}] \\ 4 \text { engines } & 0.224 \\ \text { Battery } & 0.176 \\ \text { Regulator } & 0.112 \\ \text { Electronics } & 0.170 \\ \text { Construction } & 0.515 \\ \text { Total: } & \mathbf{1 . 0 9 6}\end{array}$

Spacing of motors is $0.6 \mathrm{~m}$ and the distance between the motor shaft and the geometrical center of the structure is $l=0.3 \mathrm{~m}$.

\subsection{Measuring the thrust}

The source of force for quadrocopter hovering in the air is motors, which via rotation of propellers at high speeds generate the thrust. Knowledge of its value is required for a proper design of the whole structure. For this purpose, before designing the project's structure, force measurement for a one motor carrier was performed. A test rig was built, where the motor could rise along the $O Z$-axis. A precise electronic dynamometer was applied axially to the contact of a spinning rotor, and thrust value in relation to the step changing speed has been determined. The reference for speed is the PWM duty cycle changed by the regulator that controls rotational velocity of the motor. The PWM signal is generated in a program, where $\mathrm{PWM}=150$ corresponds to the duty cycle $\mathrm{W}=0(0 \%)$, and $\mathrm{PWM}=55$ corresponds to $\mathrm{W}=95(100 \%)$.

For example calculation (see the 14-th attempt on the graph in figure 5) of the PWM duty cycle in the non-dimensional units (nu) is as follows:

$$
W=150-\mathrm{PWM}=150-85=65[\mathrm{nu}] .
$$

In percentage it is realised as:

$W_{\%}=\frac{150-\mathrm{PWM}}{95} \cdot 100 \%=\frac{150-85}{95} \cdot 100 \%=68,4[\%]$.

According to the relation between thrust $T$ and square rotational velocity of the motor:

$$
T=b \cdot \Omega^{2},
$$

where $b$ is lifting coefficient, the dependency between the force and duty cycle $W$ could be written as follows:

$$
T=B \cdot W^{2} .
$$

After rearrangement, the coefficient of proportional dependency between the thrust and the duty cycle is obtained:

$$
B=\frac{T}{W^{2}}=\frac{7 \mathrm{~N}}{(65 \mathrm{nu})^{2}}=1.657 \cdot 10^{-3}\left[\frac{\mathrm{N}}{\mathrm{nu}^{2}}\right] .
$$

Assuming that the maximum duty cycle $W=95 \mathrm{nu}$ corresponds to the angular velocity of the propeller $\omega_{\max -\mathrm{p}}=759 \mathrm{rev} / \mathrm{min}$, the lifting coefficient $b\left[\mathrm{~N} \cdot \mathrm{s}^{2}\right]$ can be calculated as follows:

$$
b=B \frac{W^{2}}{\omega_{\max -\mathrm{p}}^{2}}=1.657 \cdot 10^{-3} \frac{\mathrm{N}}{\mathrm{nu}^{2}} \cdot \frac{(95 \mathrm{nu})^{2}}{\left(759 \frac{\mathrm{rad}}{\mathrm{s}}\right)^{2}}=2.6 \cdot 10^{-5} .
$$

Moreover, the coefficient of the moment of rotation that is taken into the numerical simulation equals:

$$
d=3.3 \cdot 10^{-5}\left[\mathrm{~N} \cdot \mathrm{m} \cdot \mathrm{s}^{2}\right] \text {. }
$$

On the basis of the measurement data, figure 5 shows a graph of thrust as a function of the PWM duty cycle in percentage values.

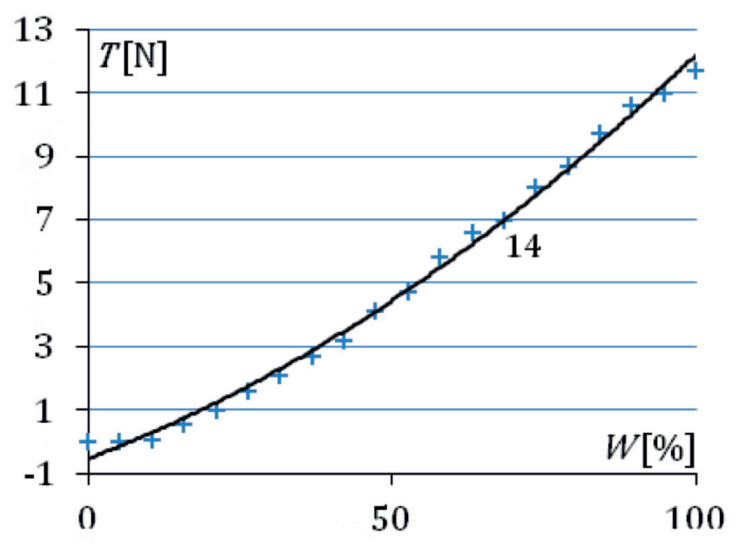

Fig. 5. Thrust versus PWM duty cycle $W_{\%}$ 


\subsection{Mathematical description of the motor}

\subsubsection{Mechanical and electrical parameters}

EMAX engine manufacturer may not describe all dynamic parameters and power of the motors, so they should be calculated as follows:

1) the supply voltage $u=11.1 \mathrm{~V}$;

2) motor constant

$$
\mathrm{k}_{\mathrm{V}}=950 \frac{\frac{\mathrm{rev}}{\mathrm{min}}}{\mathrm{V}}=950 \frac{\frac{2 \pi \mathrm{rad}}{60 \mathrm{~s}}}{\mathrm{~V}}=99.5 \frac{\mathrm{rad}}{\mathrm{V} \cdot \mathrm{s}}
$$

3) armature resistance $R=0.165 \Omega$;

4) efficiency of the rotor-propeller torque ratio $\eta=75 \%$;

5) maximum rotational velocity of the propeller $\omega_{\max -\mathrm{p}}=7250 \frac{\mathrm{rev}}{\mathrm{min}}=7250 \cdot \frac{2 \pi \mathrm{rad}}{60 \mathrm{~s}}=759 \frac{\mathrm{rad}}{\mathrm{s}} ;$

6) maximum rotational velocity of motor's shaft $\omega_{\max -\mathrm{m}}=u \cdot k_{V}=11.1 \mathrm{~V} \cdot 950 \frac{\mathrm{rev}}{\mathrm{V} \cdot \min }=10545\left[\frac{\mathrm{rev}}{\mathrm{min}}\right]$;

7) back emf (electrical) constant

$$
B=\frac{T}{W^{2}}=\frac{7 \mathrm{~N}}{(65 \mathrm{nu})^{2}}=1.657 \cdot 10^{-3}\left[\frac{\mathrm{N}}{\mathrm{nu}^{2}}\right]
$$

8) motor torque (mechanical) constant

$$
k_{m}=k_{e}=0.01\left[\frac{\mathrm{Nm}}{\mathrm{A}}\right] \text {. }
$$

The reference rotational velocity of each rotor would require additional equipment in order to perform a precise measurement after the hovering of the object at a fixed altitude, but we are able to calculate it as follows with a satisfactory accuracy.

Thrust allowing the aerial vehicle to hover in the air is $T=\frac{m g}{4}$, and from the definition $T=b \omega^{2}$; therefore, $\omega_{o}=\sqrt{\frac{m g}{4 b}}=\sqrt{\frac{1.096 \mathrm{~kg} \cdot 9.81 \frac{m}{s^{2}}}{4 \cdot 2.6 \cdot 10^{-5} \mathrm{~N} \cdot \mathrm{s}^{2}}}=321.5\left[\frac{\mathrm{rad}}{\mathrm{s}}\right]$.

At the reference rotational velocity determined by Eq. (10), the quadrocopter does not change its flight height (perfect hovering is expected).

Employing Eq. (6), the reference PWM duty cycle corresponding to $\omega_{0}$ is determined accordingly:

$$
W_{o}=\sqrt{\frac{m g}{4 B}}=\sqrt{\frac{1,096 \mathrm{~kg} \cdot 9.81 \frac{\mathrm{m}}{\mathrm{s}^{2}}}{4 \cdot 1.657 \cdot 10^{-3} \frac{\mathrm{N}}{\mathrm{nu}^{2}}}}=40[\mathrm{nu}] .
$$

The above indicated mechanical and electrical parameters will be required to derive the Laplace transfer function of the motor.

\subsubsection{Mathematical model of the motor}

The equations of motor dynamics are as follows:

$$
\left\{\begin{array}{l}
L \frac{d i}{d t}+R i+k_{e} \frac{d \theta_{m}}{d t}=u \\
I_{m} \frac{d^{2} \theta_{m}}{d t^{2}}=M_{o}-M_{l},
\end{array},\right.
$$

where $L$ indicates armature winding inductance; $R$ armature winding resistance; $k_{e}$ - back emf constant; $u(t)$ - applied voltage; $i(t)$ - motor current; $\theta_{m}(t)-$ angle of rotation of the motor's shaft; $I_{m}$ - moment of inertia reduced to the motor's shaft; $M_{o}$ - motor output torque; $M_{l}$ - motor loading torque.

The system of differential equations can be transformed to a known Laplace transfer function with the use of standard procedures:

$$
G(s)=\frac{\Omega_{m}}{U}=\frac{\frac{\frac{k_{m}}{R I_{R}}}{\frac{k_{m}^{2}}{R I_{R}}+\frac{d \omega_{o}}{\eta r^{3} I_{R}}}}{\frac{1}{\frac{k_{m}^{2}}{R I_{R}}+\frac{d \omega_{o}}{\eta r^{3} I_{R}}}+1}=\frac{k}{\tau s+1},
$$

where $\eta$ is the efficiency of the rotor-propeller torque ratio and $r$ is the ratio between the angular velocities of the motor's shaft and the propeller. After substitution of all values in Eq. (13), the first order inertial element has a gain constant of $k=11.66$ and the time delay constant of $\tau=0.004$.

\section{Mathematical modelling and motion analysis}

The quadrocopter is a type of a flying machine consisting of a rigid frame on which four propellers are mounted symmetrically. Each of them is driven by a high-speed DC motor (up to $10000 \mathrm{rev} / \mathrm{min}$ ) producing the thrust which allows to keep the object airborne.

\subsection{Equations of motion}

The flying object has two propellers that rotate clockwise and two propellers rotating counter-clockwise (Fig. 6).

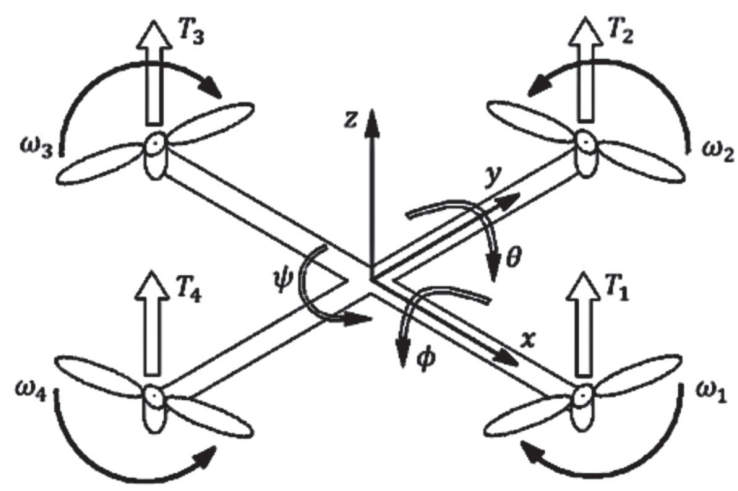

Fig. 6. Vector analysis of the quadrocopter 
This results in balancing of both torques originating from one or the other pair of propellers and control of the inclination. Each propeller produces a thrust. By changing a particular one $T_{1}, T_{2}, T_{3}, T_{4}$, or a pair of thrusts by increasing (reducing) the speed of the corresponding motor it is possible to maneuver the aerial vehicle.

The quadrocopter is characterized by 6 degrees of freedom in space: translations along $x, y, z$ axes and the rotations about $\phi, \theta, \psi$.

$$
\begin{aligned}
& I_{x x} \ddot{\phi}=\dot{\theta} \dot{\psi}\left(I_{y y}-I_{z z}\right)-I_{R} \dot{\theta} \Omega_{\mathrm{R}}+\mathrm{bl}\left(\Omega_{2}^{2}-\Omega_{4}^{2}\right) ; \\
& I_{y y} \ddot{\theta}=\dot{\phi} \dot{\psi}\left(I_{z z}-I_{x x}\right)+I_{R} \dot{\phi} \Omega_{\mathrm{R}}+\mathrm{bl}\left(\Omega_{3}^{2}-\Omega_{1}^{2}\right) ; \\
& I_{z z} \ddot{\psi}=\dot{\theta} \dot{\phi}\left(I_{x x}-I_{y y}\right)+d\left(\Omega_{1}^{2}+\Omega_{3}^{2}-\Omega_{2}^{2}-\Omega_{4}^{2}\right) ; \\
& m \ddot{x}=(\sin \phi \sin \psi+\cos \phi \sin \theta \cos \psi) T ; \\
& m \ddot{y}=(\cos \phi \sin \theta \sin \psi-\sin \phi \cos \psi) T ; \\
& m \ddot{z}=(\cos \phi \cos \theta) T-m g,
\end{aligned}
$$

where $I_{x x}, I_{y y}, I_{z z}$ indicate moments of inertia with respect to the axes $O X, O Y, O Z ; I_{R}-$ moment of inertia of the rotor; $\Omega_{i}$ - rotational velocity of $i^{\text {th }}$ rotor $(i=1 \ldots 4)$; $\Omega_{R}$ - the total rotational velocity of the rotors; $l$ - distance between the $\mathrm{OZ}$-axis and the axis passing through the centre of the propeller, the total thrust $T=T_{1}+T_{2}+$ $T_{3}+T_{4}, T_{i}-$ the particular thrust produced by the $i^{\text {th }}$ propeller; $b$ - lifting coefficient $\left[\mathrm{N} \cdot \mathrm{s}^{2}\right] ; d$ - torque coefficient $\left[\mathrm{N} \cdot \mathrm{m} \cdot \mathrm{s}^{2}\right] ; m-$ mass of the quadrocopter $[\mathrm{kg}]$; $g-$ acceleration due to gravity $\left[\mathrm{m} / \mathrm{s}^{2}\right]$.

Vector analysis of forces at uniform and non-uniform (intended) distribution of angular velocities of rotors was performed according to various tasks.

\subsection{Vector analysis of forces}

The basic tasks to be undertaken by the quadrocopter are:

- maintaining current position;

- vertical flight up and down;

- horizontal displacement along the $O X$-axis;

- horizontal displacement along the OY-axis;

- any horizontal displacement;

- left rotation about the $O Z$-axis;

- right rotation about the $O Z$-axis.

\subsubsection{Maintaining the position, lifting and lowering}

The basic task of flight is described by the equation of motion:

$$
m \ddot{z}=\cos \phi \cos \theta\left(T_{1}+T_{2}+T_{3}+T_{4}\right)-m g .
$$

For the quadrocopter to hover in the air, the thrust (a lifting force) must counteract the force of gravity, i.e.

$$
\cos \phi \cos \theta\left(T_{1}+T_{2}+T_{3}+T_{4}\right)=m g .
$$

Assuming that the machine does not move in the horizontal plane, the condition follows:

$$
T_{1}=T_{2}=T_{3}=T_{4} .
$$

With these conditions (22), the rotation does not occur $(\phi=\theta=0$, gives $\cos \phi=\cos \theta=1)$, hence:

$$
\left(T_{1}+T_{2}+T_{3}+T_{4}\right)=T=m g .
$$

Therefore, each of the propellers has to generate thrust:

$$
T_{i}=\frac{\mathrm{mg}}{4}, i=1 \ldots 4 .
$$

The aerial vehicle will rise up when the sum of lifting forces will be greater than the force of gravity, $T>m g$, and will lower flight if $T<m g$.

\subsubsection{Horizontal displacement and rotations}

Considering the movement along the $O X$-axis we should note, that the object is moved horizontally by small changes in the angles. To move the quadrocopter along the $O X$-axis, one needs to rotate it around the $O Y$-axis with a small angle $\theta$. Other angles are: $\phi=\psi=0$, i.e. $\sin \theta \neq 0$, and $\sin \phi=\sin \psi=0, \cos \phi=\cos \psi=1$, hence the following linear equations of motion are obtained:

$$
\begin{aligned}
& m \ddot{x}=\sin \theta\left(T_{1}+T_{2}+T_{3}+T_{4}\right) ; \\
& m \ddot{y}=0 ; \\
& m \ddot{z}=\cos \theta\left(T_{1}+T_{2}+T_{3}+T_{4}\right)-m g .
\end{aligned}
$$

It is worth noting, that the change in angle $\theta$ reduces influence of thrust on the quadrocopter's stable position in the air, but the influence becomes negligible when operating only at low angles. According to Eq. (21), the controlled displacement along the $O X$-axis will be achieved by increasing the thrust generated by rotor 3 , while reducing the thrust from rotor 1 .

Similarly, the controlled displacement along the $O Y$-axis will be achieved by increasing thrust $T_{4}$ generated by rotor 4 , while reducing thrust $T_{2}$. To move the object simultaneously along the $O X$ and $O Y$-axis, an increased thrust of rotors 4 and 3 has to be generated, while decreasing the thrust from rotors 2 and 1.

Implementation of rotation about the $O Z$-axis is performed by changing angle $\psi$ (yaw), which is responsible for inclination (Eq. 16). The controlled rotation to the left about the OZ-axis (counter-clockwise) will be achieved by increasing the rotational velocities $\Omega_{1}$ and $\Omega_{3}$ of rotors 1 and 3 (both rotating to the right) and decreasing at the same time the rotational velocities $\Omega_{2}$ and 
$\Omega_{4}$ of rotors 2 and 4 (both rotating to the left). During the change of all lifting forces, the total thrust $T=$ const . Rotation to the right is realized in an opposite way.

\section{Numerical simulation}

The efficiency of any mathematical model which will be used in a real control system has to be checked in numerical simulations. Thanks to the important stage of system design we are able to check if our assumptions about any parameters of the model and its dynamics are correct, and whether the expected results are achievable.

Equations (14)-(19) are rewritten in a form of 12 first order differential equations. They are well prepared in order to build the numerical model of the dynamical system:

$$
\dot{\bar{X}}=f(\bar{X}, \bar{T})=
$$

$$
\left[\begin{array}{c}
\dot{x}_{1}=\dot{\phi} \\
\dot{x}_{2}=\ddot{\phi}=\dot{\theta} \dot{\psi} \frac{\left(I_{y y}-I_{z z}\right)}{I_{x x}}-\frac{I_{R}}{I_{x x}} \dot{\theta} \Omega_{\mathrm{R}}+\frac{1}{I_{x x}} T_{1} \\
\dot{x}_{3}=\dot{\theta} \\
\dot{x}_{4}=\ddot{\theta}=\dot{\phi} \dot{\psi} \frac{\left(I_{z z}-I_{x x}\right)}{I_{y y}}+\frac{I_{R}}{I_{y y}} \dot{\phi} \Omega_{\mathrm{R}}+\frac{1}{I_{y y}} T_{2} \\
\dot{x}_{5}=\dot{\psi} \\
\dot{x}_{6}=\ddot{\psi}=\dot{\theta} \dot{\phi} \frac{\left(I_{x x}-I_{y y}\right)}{I_{z z}}+\frac{1}{I_{z z}} T_{3} \\
\dot{x}_{7}=\dot{x} \\
\ddot{x}=\frac{1}{m}(\sin \phi \sin \psi+\cos \phi \sin \theta \cos \psi) T_{4} \\
\dot{x}_{9}=\dot{y} \\
\dot{x}_{10}=\ddot{y}=\frac{1}{m}(\cos \phi \sin \theta \sin \psi-\sin \phi \cos \psi) T_{4} \\
\dot{x}_{11}=\dot{z} \\
\dot{x}_{12}=\ddot{z}=\frac{1}{m} \cos \phi \cos \theta T_{4}-g
\end{array}\right],
$$

where $\bar{X}=\left[x_{1} \ldots x_{12}\right]^{T}$ and $\bar{T}=\left[T_{1} \ldots T_{4}\right]^{T}$, elements of which are determined by Eqs (32)-(35).

Matlab Simulink has been applied to conduct the numerical simulations. On the basis of optimal dynamic responses of the analysed dynamical model, it is assumed in the control system that: $K_{p}=2$ and $K_{d}=1$, as well as all parameters introduced in Section 3. Two exemplifying tasks have been selected to show the results of simulations:

1) rotation about OX-axis by an angle $\phi=1^{\circ}$;

2) rotation about $O X$-axis by angles $\theta=1^{\circ}$ and $-1^{\mathrm{o}}, 0$.

Results of the numerical simulations which take into account the two enumerated cases have been illustrated in figures 7-16.

\subsection{Rotation about the $O X$-axis by an angle $\phi=1^{\circ}$}

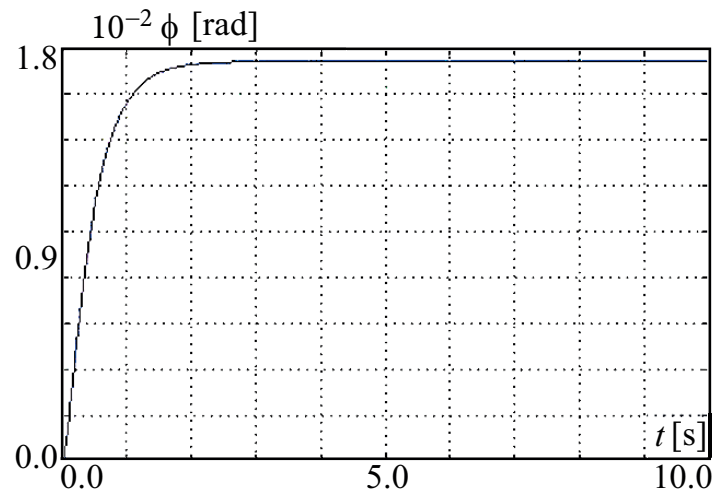

Fig. 7. Angle of rotation $\phi(t)$ about the $O X$-axis

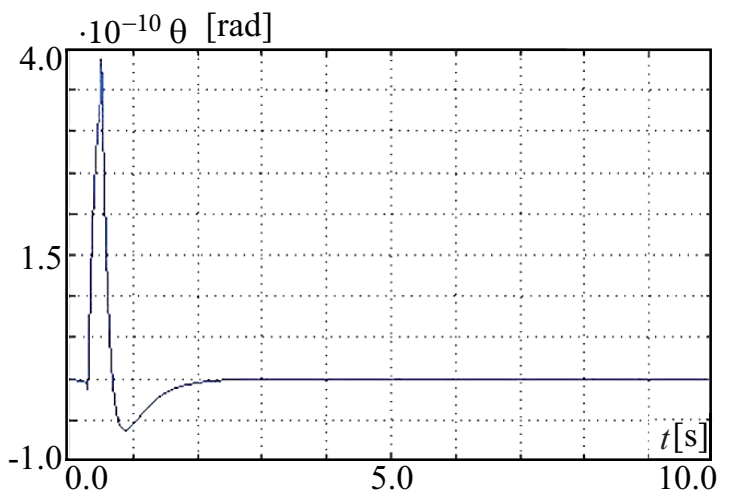

Fig. 8. Angle of rotation $\theta(t)$ about the $O Y$-axis

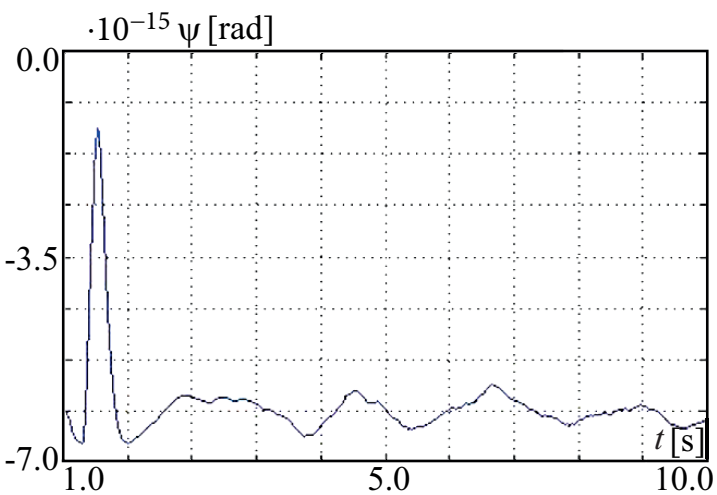

Fig. 9. Angle of rotation $\psi(t)$ about the $O Z$-axis

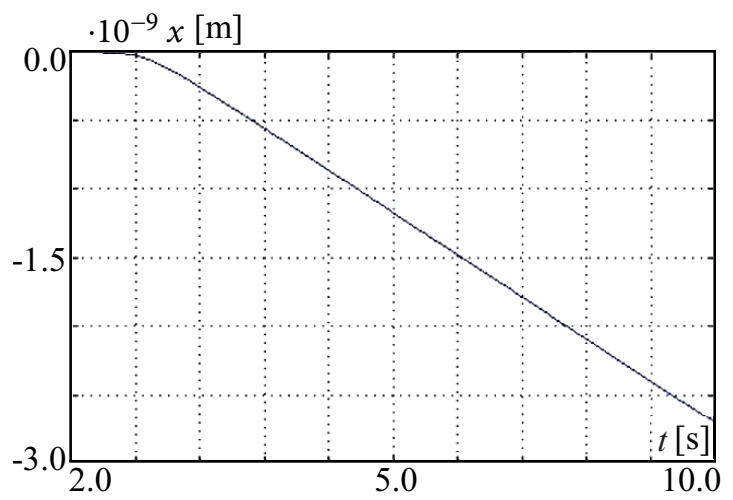

Fig. 10. Displacement along the $O X$-axis 


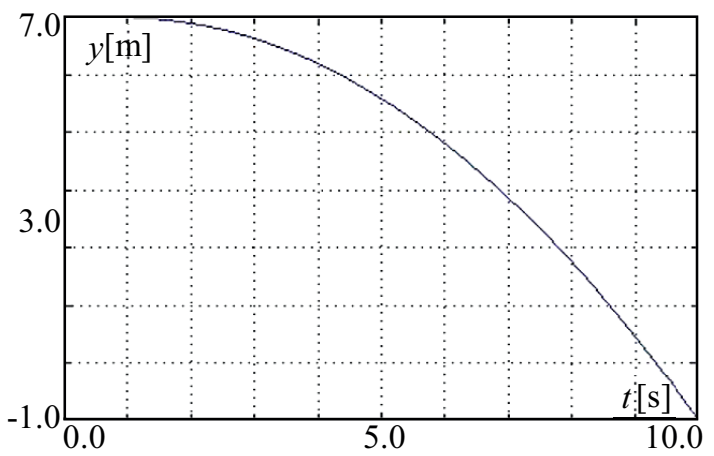

Fig. 11. Displacement along the $O Y$-axis

When setting the angle at $\varphi=1^{\circ}(0.0174 \mathrm{rad})$, the object stabilizes at the coordinate within $2 \mathrm{~s}$ (Fig. 6). Although $\theta=\psi=0$, small rotations about the $O Y$-axis and the $O Z$-axis appear because they depend on the angular velocity, $\dot{\phi}$. Besides, this dependency confirms that the unmanned aerial vehicle is very unstable. Even a temporary existence of these close to zero angles causes a displacement along the $O X$-axis. Pursuing the analysis described in Section 3.2.2, a rotation about the $O X$-axis causes some significant displacement along the $O Y$-axis.

5.2. Rotation about the $O X$-axis by angles $\theta=1^{\circ},-1,0$

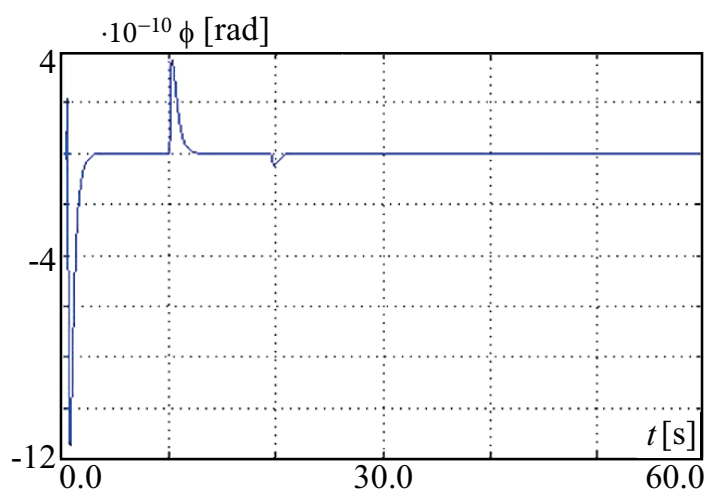

Fig. 12. Angle of rotation $\phi(t)$ about the $O X$-axis

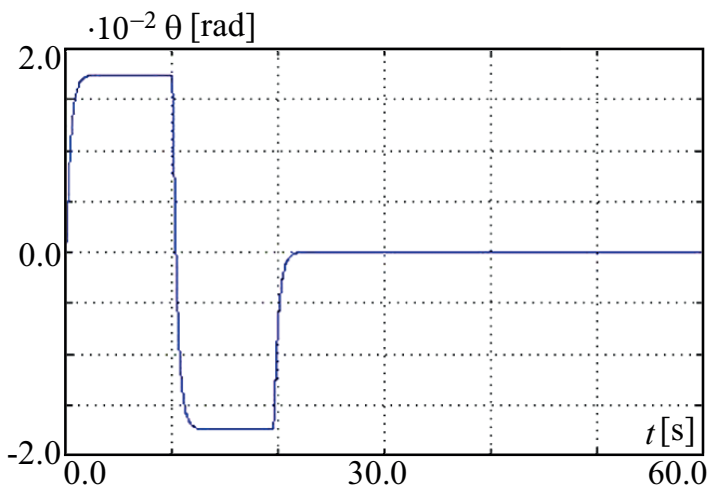

Fig. 13. Angle of rotation $\theta(t)$ about the $O Y$-axis

Similarly to the previous case (Fig. 11), the rotation angles $\phi$ and $\psi$ vary slightly in time causing a small displacement along the $O Y$-axis. The object has been accelerated along the $O X$-axis, and then slowed down to assume a particular position.

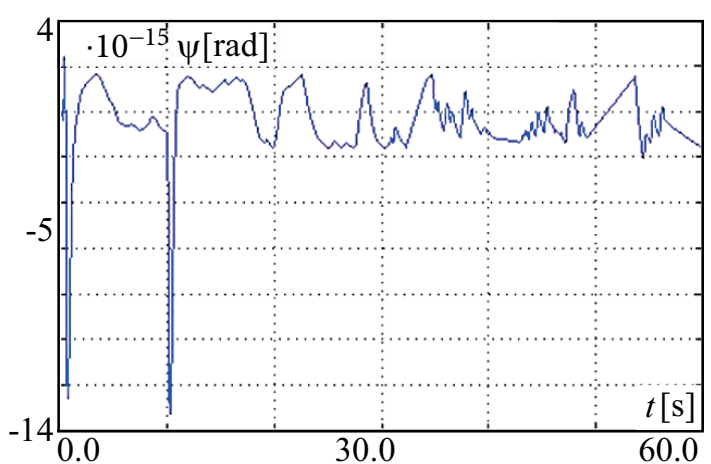

Fig. 14. Angle of rotation $\psi(t)$ about the $O Z$-axis

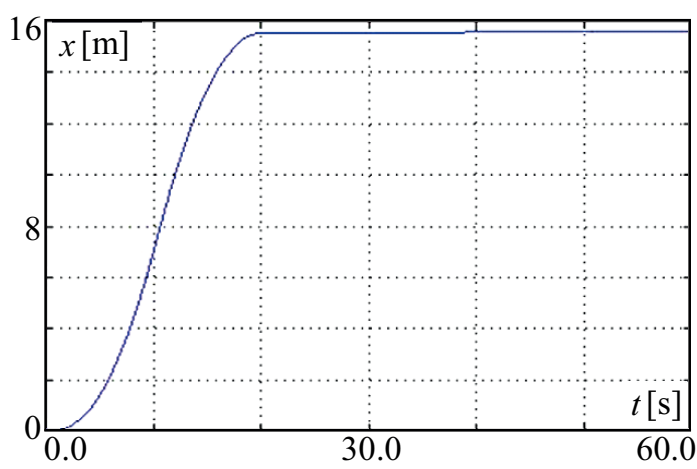

Fig. 15. Displacement along the $O X$-axis

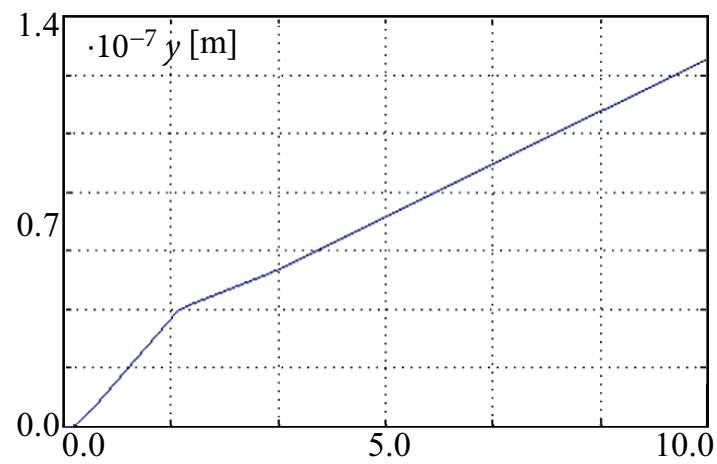

Fig. 16. Displacement along the $O Y$-axis

\section{Control of the UAV}

\subsection{Theoretical background}

The quadrocopter is an object difficult to control, because its weight and constants of time delays are small. Building the control system we must identify the input and output signals passing through the object of control.

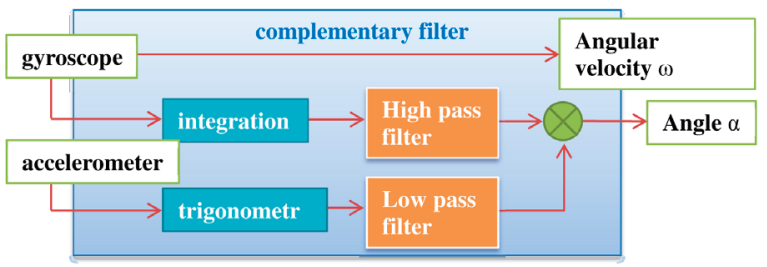

Fig. 17. The general system of data acquisition 
Signals representing the three angles of rotation and the corresponding speed that are received from the accelerometer and gyroscope will create the measured system states. The system will react to the changes by controlling excitations generated by force $U_{4}$ and the moments $U_{1}, U_{2}, U_{3}$.

The complementary filter shown in figure 17 is described by the recursive formula:

$$
\alpha_{n}=(1-x)\left(\alpha_{n-1}+\dot{\alpha} d t\right)+x \tilde{\alpha}_{n},
$$

where $\alpha_{n}$ indicates the filtered variables $(\phi, \theta$ or $\psi)$ in $n$-th measurement; $x$ - non-dimensional constant of the filter; $d t$ - a time interval between successive measurements (a sampling period); $\tilde{\alpha}_{n}$ - value of the variable read from the input ports of a gyroscope (measurement of rotational velocity) or an accelerometer (measurement of the angles of rotation).

The most important data for the control of the quadrocopter are the current angles of rotation around axes: $\phi_{n}, \theta_{n}, \psi_{n}$ and the corresponding angular velocities: $\omega_{\phi n}=\dot{\phi}, \omega_{\theta n}=\dot{\theta}$ and $\omega_{\psi n}=\dot{\psi}$. These variables being the inputs to the Proportional Integral (P-D) controller of the form are used to compute errors of regulation:

$$
\begin{aligned}
& \tilde{T}_{1}=I_{x x}\left(K_{p}\left(\bar{\phi}_{r}-\phi_{n}\right)+K_{d}\left(\bar{\omega}_{\phi}-\omega_{\phi n}\right)\right) ; \\
& \tilde{T}_{2}=I_{y y}\left(K_{p}\left(\bar{\theta}_{r}-\theta_{n}\right)+K_{d}\left(\bar{\omega}_{\theta}-\omega_{\theta n}\right)\right) \\
& \tilde{T}_{3}=I_{z z}\left(K_{p}\left(\bar{\psi}_{r}-\psi_{n}\right)+K_{d}\left(\bar{\omega}_{\psi}-\omega_{\psi n}\right)\right) .
\end{aligned}
$$

Torques, $\tilde{T}_{i}$, are responsible for the excitation of the resultant Quadrotor's rotation around axes $O X, O Y, O Z$. One assumes that after the action, the controlled flying object (a plant) will take the desired state. On the other hand, the required torques, $T_{i}$, can be calculated from Eqs (20)-(22) using the following simplified relations:

$$
\begin{aligned}
& T_{1}=b l\left(\Omega_{2}^{2}-\Omega_{4}^{2}\right) ; \\
& T_{2}=b l\left(\Omega_{3}^{2}-\Omega_{1}^{2}\right) ; \\
& T_{3}=d\left(\Omega_{1}^{2}+\Omega_{3}^{2}-\Omega_{2}^{2}-\Omega_{4}^{2}\right) ; \\
& T_{4}=b\left(\Omega_{1}^{2}+\Omega_{3}^{2}+\Omega_{2}^{2}+\Omega_{4}^{2}\right) .
\end{aligned}
$$

When comparing Eqs (30)-(31) with (33)-(34), four angular velocities needed to drive the quad rotor mobile object can be calculated from the formulas:

$$
\begin{aligned}
& \Omega_{1}^{2}=\Omega_{n}^{2}-\frac{\tilde{T}_{2}}{2 b l}+\frac{\tilde{T}_{3}}{4 d} ; \\
& \Omega_{2}^{2}=\Omega_{n}^{2}+\frac{\tilde{T}_{1}}{2 b l}-\frac{\tilde{T}_{3}}{4 d} ; \\
& \Omega_{3}^{2}=\Omega_{n}^{2}+\frac{\tilde{T}_{-} 2}{2 b l}+\frac{\tilde{T}_{3}}{4 d} ; \\
& \Omega_{4}^{2}=\Omega_{n}^{2}-\frac{\tilde{T}_{1}}{2 b l}-\frac{\tilde{T}_{3}}{4 d} .
\end{aligned}
$$

Altitude of the quadrocopter is set manually by assigning a specific angular velocity, $\Omega_{n}$. One could determine the angular velocities of each motor according to the torque caused by the errors of regulation.

\subsection{Design and components of the control unit}

In figure 18, a schematic view of main connections between components of the quadrocopter's control unit are presented.

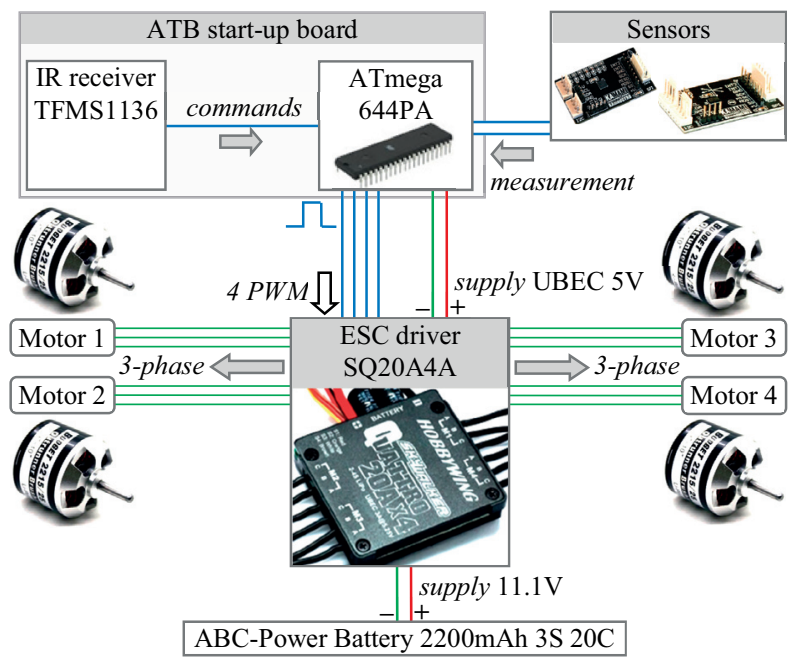

Fig. 18. A schematic view of the connections between main components of the control unit

List of components:

- start-up board ATB ATMEGA644P with ISP connector and IR receiver TFMS1136;

- 4 x motor Budget 2215/25 KV950-185W;

- 2 x propeller AEO 10x4, 10x4 7RSF+, 7SF;

- ABC-Power LiPo Battery 2200mAh 3S 20C;

- charger with balancer IMAX B3-2S/3S;

- controller ESC Hobbywing Skywalker QuattroSQ20A4U;

- KAmodMEMS2 3-axis accelerometer LIS35DE;

- KAmod GYRO 3-axis gyroscope L3G4200d.

The flying object is controlled by a microcontroller located on the ATB start-up board.

Commands are sent by pressing the appropriate buttons on the remote control supplied with the board. The signals informing about the current Quadrotor's state are retrieved from an accelerometer and a gyroscope. The control unit processes the data and then sends specific duty cycles that are transferred to the rotational velocities of four three-phase BLDC motors. The motors are sourced by the ESC driver, which is powered by an $\mathrm{ABC}$-Power LiPo battery with a nominal voltage of 11.1V. The ESC combines four high performance $20 \mathrm{~A}$ ESCs and a $5 \mathrm{~V} / 3 \mathrm{~A}$ UBEC, where the ESC is based on standard PWM control signal. By means of the $5 \mathrm{~V} / 3 \mathrm{~A}$ UBECs' other components, such as the microcontroller, infrared receiver and sensors are powered. 


\section{Conclusions}

On the basis of mechanical and dynamical analyses, an unmanned aerial vehicle has been designed and built. The object is able to hover in the air.

The stress analysis and testing of a number of possible structures has led to the design of a prototype object.

The derived differential equations describing the dynamics of motion of the object have enabled us to propose the mathematical form of a PD controller based on the signals processed by a complementary filter.

Preliminarily selected ATB board with ATmega 644PA microcontroller was partially functional, because it allows transmitting the control signals only via an IR receiver.

The quadrocopter, as a result of the described work, fulfills many requirements and simultaneously allows for development of the construction in the future. The construction has been assembled from light but durable materials, which are essential in constructing any flying machines.

Currently, we are improving electronics and programming of the processor computing the voltages required to source all motors in real-time. This will allow performing more complex flight tasks. The authors intend to improve the communication, stabilization and will try to mount a video camera.

\section{Acknowledgements}

The authors have been supported by the National Center of Science under the grant MAESTRO 2, No. 2012/04/A/ ST8/00738 in the period 2012-2015 (Poland).

\section{References}

Alexis, K.; Nikolakopoulos, G.; Tzes, A. 2012. Model predictive quadrotor control: attitude, altitude and position experimental studies, IET Control Theory \& Applications 6(15): 1812-1827. http://dx.doi.org/10.1049/iet-cta.2011.0348

Bi, Y.; Duan, H. 2013. Implementation of autonomous visual tracking and landing for a low-cost quadrotor, Optik - International Journal for Light and Electron Optics 124(18): 3296-3300.

Gupte, S.; Mohandas, P. I. T.; Conrad, J. M. 2012. A survey of quadrotor Unmanned Aerial Vehicles, in Southeastcon, 2012 Proceedings of IEEE, 15-18 March, 2012, Orlando, 1-6. http://dx.doi.org/10.1109/SECon.2012.6196930

Hoffmann, G. M.; Huang, H.; Waslander, S. L., et al. 2011. Precision flight control for a multi-vehicle quadrotor helicopter testbed, Control Engineering Practice 19(9): 10231036. http://dx.doi.org/10.1016/j.conengprac.2011.04.005

Minh, L. D.; Ha, C. 2010. Modeling and control of quadrotor MAV using vision-based measurement, in 2010 International Forum on Strategic Technology IFOST, 13-15 October, 2010, Ulsan, 70-75. http://dx.doi.org/10.1109/IFOST.2010.5668079
Mohammadi, M.; Mohammad Shahri, A. 2013. Modeling and decentralized adaptive tracking control of a quadrotor UAV, in 2013 First RSI/ISM International Conference on Robotics and Mechatronics (ICRoM), 13-15 February, 2013, Tehran, 293-300. http://dx.doi.org/10.1109/ICRoM.2013.6510122

Nicol, C.; Macnab, C. J. B.; Ramirez-Serano, A. 2011. Robust adaptive control of a quadrotor helicopter, Mechatronics 21(6): 927-938.

http://dx.doi.org/10.1016/j.mechatronics.2011.02.007

Olejnik, P.; Awrejcewicz, J. 2013. Low-speed voltage-input tracking control of a DC-motor numerically modelled by a dynamical system with stick-slip friction, Differential Equations and Dynamical Systems 21(1-2): 3-13. http://dx.doi.org/10.1007/s12591-012-0114-x

Raffo, G. V.; Ortega, M. G.; Rubio, F. R. 2011. Path tracking of a UAV via an underactuated $\mathrm{H}$ control strategy, European Journal of Control 17(2): 194-213. http://dx.doi.org/10.3166/ejc.17.194-213

Runcharoon, K.; Srichatrapimuk, V. 2013. Sliding mode control of quadrotor, in Electronics and Computer Engineering (TAEECE), 2013 International Conference on Technological Advances in Electrical, 9-11 May, 2013, Konya, Turkey, 552-557.

Ryll, M.; Bulthoff, H. H.; Giordano, P. R. 2012. Modeling and control of a quadrotor UAV with tilting propellers, in 2012 IEEE International Conference on Robotics and Automation (ICRA), 14-18 May, 2012, Saint Paul, 4606-4613. http://dx.doi.org/10.1109/ICRA.2012.6225129

Sahoo, D.; Kumar, A.; Sujatha, K. 2010. A survey on remotely operated quadrotor aerial vehicle using the camera perspective, International Journal of Computer Applications 11(10): 1-6.

Senkul, F.; Altug, E. 2013. Modeling and control of a novel tiltroll rotor quadrotor UAV, in 2013 International Conference on Unmanned Aircraft Systems (ICUAS), 28-31 May, 2013, Atlanta, 1071-1076.

Yali, Y.; Feng, S.; Yuanxi, W. 2012. Controller design of quadrotor aerial robot, in 2012 International Conference on Medical Physics and Biomedical Engineering (ICMPBE2012), Physics Procedia 33(0): 1254-1260.

Zhang, T.; Li, W.; Achtelik, M., et al. 2011. Multi-Sensory motion estimation and control of an autonomous quadrotor, Advanced Robotics 25(11-12): 1493-1514. http://dx.doi.org/10.1163/016918611X579501 